\title{
Recency Tendency: Responses to Forced-Choice Questions
}

\author{
MEHDI B. MEHRANI ${ }^{1} *$ and CAROLE PETERSON ${ }^{2}$ \\ ${ }^{1}$ English Department, University of Neyshabur, Neyshabur, Iran \\ ${ }^{2}$ Department of Psychology, Memorial University of Newfoundland, Canada
}

\begin{abstract}
Summary: The present study was conducted to investigate whether forced-choice questions would lead to any particular tendency in young children's responses. Two experiments were conducted in which 3-to 5-year-olds children were shown a short animation and then were asked a set of two-option, forced-choice questions. Consistent findings were obtained: (i) Forced-choice questions influenced children's responses; (ii) Children displayed a consistent 'recency tendency.' That is, they tended to choose the second option in forced-choice questions; (iii) This tendency grew weaker as children aged. The findings suggest that forced-choice questions carry some suggestibility load and can bias children's responses. Copyright @ 2015 John Wiley \& Sons, Ltd.
\end{abstract}

In developmental psychology, questioning is used as an important method for obtaining data from children (Fritzley \& Lee, 2003). In medical settings, it is employed as the most immediate available method for measuring the intensity and other features of pain. In forensic investigations, particularly in the case of children's sexual abuse, questioning is not only important, it is sometimes considered to be the only source of information (because sexual abuse is often secretive). However, many developmental psychologists have expressed concerns about the reliability of children's responses to adults' questions (Ceci \& Bruck, 2006; Howie, Kurukulasuriya, Nash, \& Marsh, 2009; Mehrani, 2011; Okanda \& Itakura, 2007, 2008, 2010; Rocha, Marche, \& Briere, 2013).

Developmental studies have witnessed increasing interest over the past two decades about the credibility of children's answers to interview questions, and this wave of interest has been reinforced by medical, cultural, legal, and psychological concerns of the day. The contributions of studies dealing with the reliability of children's responses are applicable to a variety of disciplines. For example, in forensic psychology, the results of these studies can hold important implications for how legal cases are decided, including whether individuals face long prison terms or are given lifetime labels as sexual offenders (Reyna, Holliday, \& Marche, 2002). In medical science, these studies provide insightful findings for children's medical and dental checkups (Peterson \& Biggs, 1997), such as whether something hurts (e.g., a tummy or a tooth). In applied linguistics and language testing, they can be used to avoid probable biases in designing and developing language tests.

In response to these theoretical and practical demands across different disciplines, vigorous academic debates have taken place about issues related to children's responses (Newcombe \& Dour, 2001). Although some studies have reported that children can provide reliable and fairly accurate information, this is particularly true when free recall or openended questions are used (Brown et al., 2013; Peterson, 2012; Quas, Goodman, Ghetti, \& Redlich, 2000). Trying to determine the most appropriate ways in which to question children is still an important goal of researchers (Brown,

*Correspondence to: Mehdi B. Mehrani, English Department, University of Neyshabur, Neyshabur, Iran.

E-mail: mehrani@neyshabur.ac.ir
Lewis, Lamb, \& Stephens, 2012; Ceci \& Bruck, 2006). In particular, many researchers have expressed concerns that young children's responses to questions, especially those that are not open-ended, may be easily influenced by a host of social and cognitive factors (Ceci \& Bruck, 2006; Goodman \& Quas, 2008; Mehrani, 2011; Mehrani \& Peterson, 2014). Consequently, researchers have investigated factors that moderate children's performance in question-answer situations.

A number of experimental studies have investigated the effects of question format on children's inconsistencies when answering repeated questions about past events. For instance, Poole and White (1991) reported that 4-year-olds (although not 6- or 8-year-olds or adults) shifted their responses to repeated yes/no questions about an ambiguous event that they had individually witnessed, but they did not do so to open-ended questions. Howie, Sheehan, Mojarrad, and Wrzesinska (2004) asked children repeated questions and compared their responses to open-ended, forcedchoice two-option, and yes/no questions. They included unbiased (open-ended and two-option) questions and misleading (all of which were yes/no) questions. In their study, 4-year-old to 5-year-old children appeared vulnerable to shifting their responses to both two-option questions and misleading yes/no questions, whereas 7- to 8-year-olds were mostly only vulnerable to misleading yes/no questions. However, questions in their study were not systematically matched for content in that yes/no questions were always misleading with a 'no' response as correct, and either of the other question formats could be paired with each misleading question, and option 1 versus 2 were never compared in two-option questions. Thus, it is difficult to make definitive conclusions about the effect of question format. Peterson and Grant (2001) also compared preschoolers' responses to yes/no and forced-choice two-option questions. Although children in their study demonstrated a tendency toward saying yes to yes/no questions, they did not exhibit any response biases for forced-choice questions, choosing the two given options equivalently often. In a different comparative study, Brown et al. (2013) investigated the accuracy of 5-year old to 7-year-old children's responses to open-ended and directive prompts (including yes/no and forced-choice questions) about experienced events. They found that open-ended prompts elicited more detailed responses than 
more focused directive prompts without reducing accuracy. In another study, Howie et al. (2009) controlled for the content of questions and reported that only 4-year-old children show evidence of sensitivity to question format. They found that, regardless of question format, 5- and 7-year olds changed their responses when adults repeated their questions, but 4-year olds were more likely to shift their response to yes/no questions whose answer was 'no' than to forced-choice two-option questions. These researchers maintained that the misleading nature of repeated questions possesses a particular problem because of perceived pressure, over and above any effect of question format. Recently, Rocha et al. (2013) compared different types of forced-choice questions in 4- to 12-year-olds about a dental visit that included two-option and yes/no questions, and they found a bias toward saying 'yes' in yes/no questions. In addition, although they did not specifically mention a recency bias in children's responses, their data are consistent with the existence of such a bias toward choosing option 2 in two-option questions in their youngest age group of 4- to 6-year-olds, although not with older children. Other aspects of their study are discussed as follows.

It is well established that preschoolers are more likely to provide false answers to questions that restrict the scope of their responses. Such questions are referred to as 'leading questions' in the literature. Goodman and Schaaf (1997) define a leading question as a question that provides information that is not already mentioned. Endres (1997) maintains that the more information there is in a question that can possibly cue a desired or expected answer, the more leading or suggestive the question is. On this basis, forced-choice two-option questions can be considered to be highly leading, because the respondent is asked to select between the options already embedded in such questions. However, systematic investigations of such forced-choice questions are rare (Peterson \& Grant, 2001; Rocha et al., 2013). As Peterson, Dowden, and Tobin (1999) assert, 'Unfortunately, much and perhaps even most research on suggestibility uses almost nothing but yes/no format questions' (p. 541). An exception is a study by Fritzley, Lindsay, and Lee (2009). They explored 2- to 5-year-old children's responses to two-option forced-choice questions, varying familiarity of the items asked about and whether the questions were irrelevant or nonsensical. They found that children of all ages strongly preferred the second option, and this recency bias was heightened when the queried objects were unfamiliar or the questions were 'problematic.' Another exception is the study carried out by Rocha et al. (2013) that was referred to earlier. In this investigation, the authors not only assessed the accuracy of children's responses to two-option multiple choice questions and yes/no questions but they also explored suggestibility effects of these two question formats. The multiple choice questions could have as the correct answer option 1, option 2, or neither stated option was correct. The yes/no questions could have as the correct answer either yes or no, or they could have queried an absent feature, that is, asked a yes/no format question about something that was not related to the target event. The authors found that question format was an important determiner of both children's accuracy and their suggestibility. Children at all ages were most inaccurate as well as most suggestible when they were given a two-option question where neither option was correct. For children in the youngest age group (4-6 years old), the second worst type of question in terms of inaccuracy and suggestibility was a yes/no question in which 'no' was the correct option. In addition, accuracy and suggestibility on two-option multiplechoice questions in which the correct response was option 1 were not significantly different from their performance on yes/no questions where 'no' was the correct response. Thus, children this young were more accurate and less suggestible when option 2 was the correct response in multiple choice questions, which is consistent with a recency bias. Overall, then, the limited literature concerning preschoolers' responses to forced-choice questions is mixed: Fritzley et al. (2009) found a strong recency bias for preschoolers, Rocha et al. (2013) found a small recency bias for preschoolers only, and Peterson and Grant (2001) found no bias.

A serious problem for interviewers is that young children do not often provide much information during free recall or after general open-ended prompts, (see Pipe, Thierry, \& Lamb, 2006 and Powell, Fisher, \& Wright, 2005 for reviews). To elicit information not provided after openended prompts, interviewers resort to other types of questions that have restricted response options and have the potential to lead or mislead the child (Larsson \& Lamb, 2009; Peterson \& Biggs, 1997; Peterson \& Grant, 2001). Researchers have reported that forced-choice questions are frequently used in various situations, including forensic contexts (Lamb et al., 2003), in spite of recommendations to avoid such questions (Lamb, Orbach, Hershkowitz, Esplin, \& Horowitz, 2007). For example, Davies, Tarrant, and Flin (2000) found that in interviews conducted by police officers in the UK with suspected child victims of sexual abuse, almost half of the questions were either yes/no or forced-choice questions. Therefore, because of the ubiquitous use and considerable importance of forcedchoice questions, the implications of asking such questions need to be understood. In the present study, two experiments were conducted to examine whether forced-choice questions influence children's responses and to clarify some of the inconsistencies in the limited literature regarding forced-choice questions. More specifically, this study investigates the effects of word order in two-option, forced-choice questions where the correct answer varies between options 1 and 2 . It also examines whether preschoolers show any tendency toward choosing either of the options.

\section{EXPERIMENT 1}

This experiment was conducted to investigate whether preschoolers of different ages display any tendency toward either of the stated options in forced-choice questions. Due to the inconsistencies in the literature, the exact direction of children's response tendencies could not be easily predicted. However, based on previous studies on other types of questions, it was hypothesized that such a tendency, if it exists, would grow weaker as children's age increases. 


\section{Participants}

The participants were 90 Iranian preschoolers, including 30 three-year-olds (14 male and 16 female, age range $=38-47$ months, $M=42.1$ months, $S D=1.91)$; 30 four-year-olds (12 male and 18 female, age range $=49-60$ months, $M=56.2$ months, $S D=4.1$ ); and 30 five-year-olds (11 male and 19 female, age range $=61-70$ months, $M=66.8$ months, $S D=2.87)$. All children were monolingual speakers of Persian, and they were recruited from three child care centers in Neyshabur, Iran. The child care centers were located in the downtown area and included preschoolers from neighborhoods where mostly middle-class, working families lived. Participants came from similar socio-economic backgrounds, minimizing possible social and cultural variations. No children in this and the subsequent experiment participated more than once in the process of data collection.

\section{Procedure and materials}

A short animation made for children was used. The animation depicted a simple story, which took place in a jungle and its characters were some animals including a rabbit, a chicken, a crow, a bear, and a monkey. Prior to the main experiment, a pilot study was conducted to ensure children that were familiar with the animals and could understand the story and the words used in the animation. A small number of children $(N=12$, ranging in age from 3- to 5-year-olds) were shown the animation and were asked questions about the story and the characters. Based on the pilot study, a forced-choice question task was designed, which included 16 two-option questions. The question sequence followed the temporal order of events in the film. Only simple and easily understandable words were used in designing the task (see Table 1 for the translated wording of the questions asked.) The correct answer to 8 out of 16 questions was the first option stated in the question stem (hereafter 'first option' questions), and for the other 8 questions, the correct answer was the second option (hereafter 'second option' questions). To counterbalance the order of the presentation of first and second option questions, two versions of the task were developed. Children were randomly assigned to one of two groups for questioning. The first group received the task, which started with a first option question followed by a second option question, followed by a first option question, and so on. The second group received the same task in the opposite direction: the first question was the second option, followed by a first option question, and so on.

During the main phase of the study, after a short rapportbuilding introduction, children were instructed on how to participate in the study. In particular, they were informed that they could say 'I don't know' if they did not know the answers. Children were then shown the animation and interviewed individually in their child care center by a research assistant who had no prior knowledge about the research design and questions. Each child's response was written on a scoresheet immediately after each question. In addition, children's responses were recorded to confirm accurate scoring. Finally, the children were thanked and rewarded with a photo book.

\section{Results}

Children's responses were scored by assigning a +1 to each correct answer to the two types of questions, first option and second option. Incorrect answers, 'I don't know responses', and 'unanswered questions' received scores of 0 . Therefore, every child had a score for the number of correct responses to both first option questions and second option questions, with a maximum score of +8 and a minimum score of zero.

First, the frequency of 'I don't know' responses and unanswered questions was investigated. Children at all ages seldom responded 'I don't know'. They also responded to almost all questions and rarely left questions unanswered. That is, there were only nine times across all children that 'I don't know' responses were observed (across a total of 1440 questions), and only seven times 'no answer' responses were observed in all age groups. To investigate the effects of age and the order of the correct option in forced-choice questions on children's responses, a 3 (age: 3-year-olds, 4-yearolds, and 5-year-olds) $\times 2$ (order of correct constituent: option 1 versus option 2) mixed-design analysis of variance was performed on children's response scores with the order of correct constituents as the repeated measure. Results indicated a significant effect for the order of correct constituents, $F(1,87)=40.45, p<.001$, and $\eta^{2}=.27$. The children exhibited a tendency toward choosing the second option of the forced-choice questions. In addition, the effect of age was significant, $F(2,87)=7.35, p=.001$, and $\eta^{2}=.08$. As age increased, children's bias toward choosing the second option grew weaker. The interaction between the order of correct constituents and age was also significant, $F(1,87)=10.11$, $p<.001$, and $\eta^{2}=.13$. Tukey post hoc comparisons were carried out separately for each option, and they revealed that the age groups did not differ when option 2 was the correct choice, but when option 1 was correct, the difference between the 3-year-olds' mean scores and those of the other age groups was significant $(p<.01)$. The 4 - and 5 -year-olds groups did not significantly differ from each other. The relevant descriptive statistics are presented in Figure 1.

Table 1. Questions asked in experiment 1

Was it winter or spring?

Were the animals in a jungle or in a zoo?

Was the chicken planning alone or with its friends?

Who went after the rabbit, the monkey or the crow?

Was the problem that the rabbit had a cold or snored?

Who could heal the rabbit, the physician or the hen?

Was the rabbit sleeping on a leaf or in its home?

Who was snoring, the rabbit or the chicken?
Did the crow say that the squirrel was coming or the bear was coming? Who visited the chicken, the monkey or the sparrow?

Was the rabbit snoring under a tree or in its home?

Was the rabbit taken to the chicken's home or to the hospital?

Who came last, the squirrel or the fox?

Did the physician make the rabbit go away or the fox go away?

Was the chicken dumb or smart?

Did the fox go away or did he eat them? 


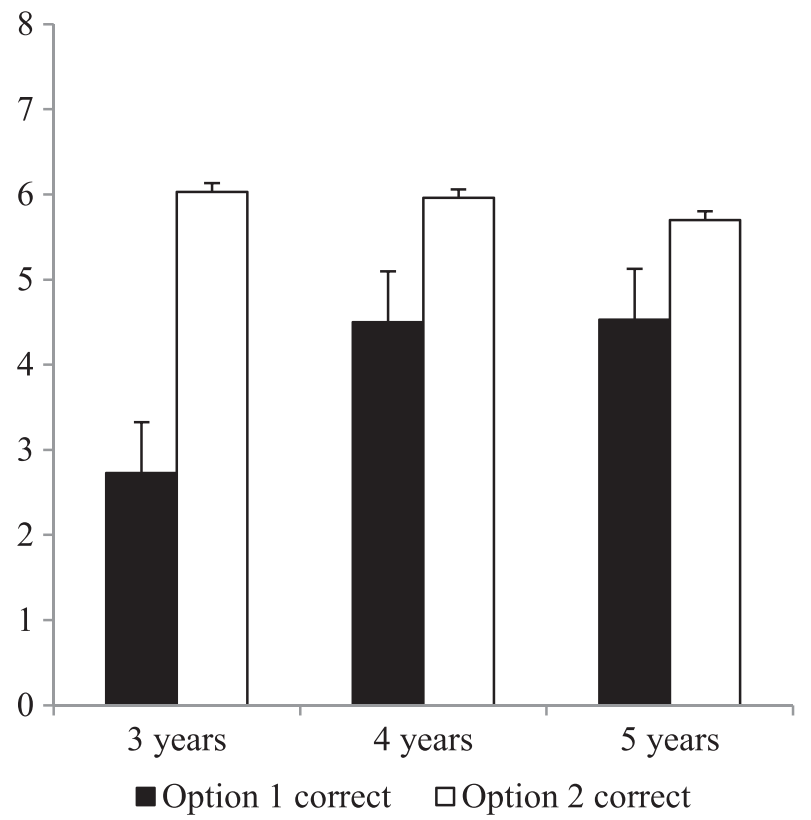

Figure 1. Experiment 1: Means for the number of correct answers as a function of age and whether the accurate response was option 1 or option 2. Standard errors are represented in the figure by the error bars attached to each column

As is shown in Figure 1, this experiment confirmed that children show a 'recency tendency' in response to forcedchoice questions. That is, children tend to choose the last options stated in forced-choice questions. In addition, the findings revealed that age had a significant effect on children's responses to two-option forced-choice questions: recency tendencies were more pronounced in 3-year-olds' responses than in 4- and 5-year-olds' responses.

\section{EXPERIMENT 2}

In experiment 2 , the researchers investigated whether the observed recency tendency in young children could be replicated. Therefore, following the principles of split ballot technique, the procedure used in experiment 1 was modified, and children were asked the same questions in the opposite direction. For instance, the question 'Was it spring or winter?' was asked as 'Was it winter or spring?'. While in response to the former question, children needed to select the first option (spring) in order to correctly answer this question; in the latter case, they were required to choose the second option. This was applied to all 16 questions (see Table 2 for a translated list of the questions asked in experiment 2). The purpose of this procedural modification was to ensure that children's response accuracy was attributed to question format, namely, whether the correct answer was option 1 versus option 2 rather than chance or the specific objects asked about. Based on the findings of experiment 1 , it was hypothesized that there would be a significant effect of word order, and younger children would display a stronger recency tendency than older children.

\section{Participants}

Like those who participated in experiment 1 , children in this experiment were monolingual speakers of Persian, and they were recruited from four child care centers. There were 24 three-yearolds (11 male and 13 female, age range $=36-47$ months, $M=43.5$ months, $S D=2.23)$; 22 four-year-olds (12 male and 10 female, age range $=48-58$ months, $M=54.6$ moths, $S D=3.1)$; and 21 five-year-olds (10 male and 11 female, age range $=62-71$ months, $M=67.5$ months, $S D=2.06$ ), for a total of 67 children. All children were from middle socioeconomic backgrounds.

\section{Procedure and materials}

The procedure and the materials used in this experiment were the same as those used in experiment 1, except for the modifications made on questions. That is, the order of the correct constituents of the questions was reversed, so that if the correct answer was the first option in Experiment 1, it was the second option in this experiment.

\section{Results}

As the explicit instruction about the permissibility of responding 'I don't know' did not increase the likelihood of this response being chosen by the children in Experiment 1, we did not expect a high frequency of 'I don't know' responses in this experiment. Children at all ages seldom responded 'I don't know.' That is, only 13 times 'I don't' know' responses were observed across all children (across 1072 questions). Also, children at all ages left only 8 questions unanswered.

Two response tendency scores were obtained for each child, using the same procedure used in experiment 1 . A 3 (age: 3-year-olds, 4-year-olds, and 5-year-olds) $\times 2$ (order of correct constituent: option 1 or option 2) mixed-design analysis of variance was conducted on children's scores with the order of constituents as the repeated measure. Results indicated a significant effect of the order of correct constituents, $F(1,64)=37$. 86, $p<.001$, and $\eta^{2}=.29$ (Figure 2). Children displayed a tendency toward the second option of the questions. In addition, the effect of age was significant,

Table 2. Questions asked in experiment 2

\section{Was it spring or winter?}

Were the animals in a zoo or in a jungle?

Was the chicken planning with its friends or alone?

Who went after the rabbit, the crow or the monkey?

Was the problem that the rabbit snored or had a cold?

Who could heal the rabbit, the hen or the physician?

Was the rabbit sleeping in its home or on a leaf?

Who was snoring, the chicken or the rabbit?
Did the crow say that the bear was coming or the squirrel was coming?

Who visited the chicken, the sparrow or the monkey?

Was the rabbit snoring in its home or under a tree?

Was the rabbit taken to the hospital or to the chicken's home?

Who came last, the fox or the squirrel?

Did the physician make the fox go away or the rabbit go away?

Was the chicken smart or dumb?

Did the fox eat them or did he go away? 


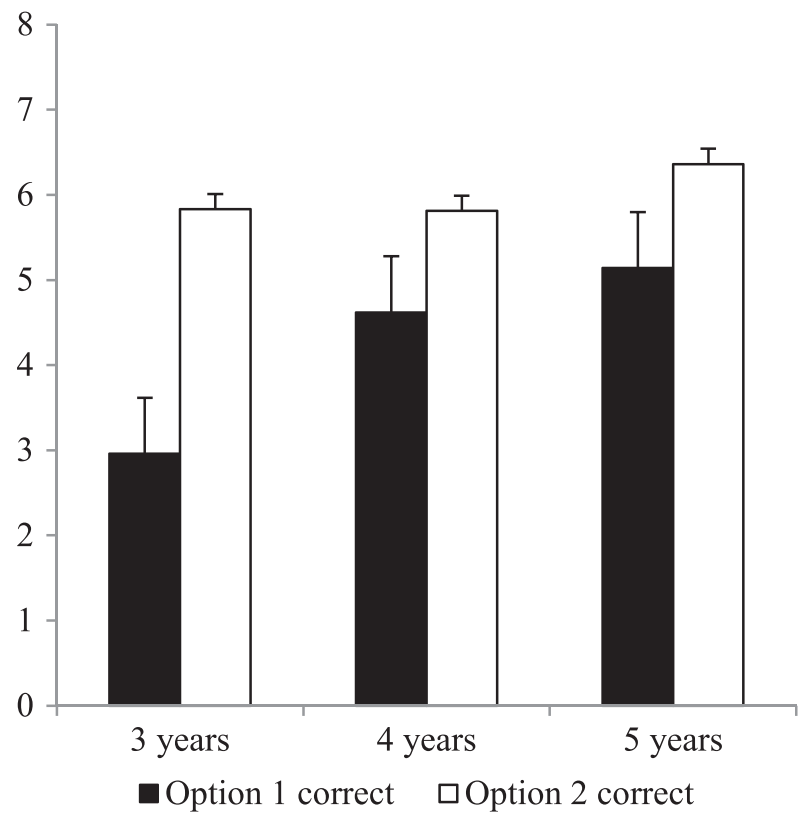

Figure 2. Experiment 2: Means for the number of correct answers as a function of age and whether the accurate response was option 1 or option 2. Standard errors are represented in the figure by the error bars attached to each column

$F(2,64)=6.90, p=.002$, and $\eta^{2}=.11$. As age increased, children's response bias toward the second option grew weaker. The interaction between the order of constituents and age was also significant, $F(1,64)=6.29, p=.003$, and $\eta^{2}=.10$. Tukey post hoc comparisons were carried out separately for each option, and they showed that for option 1 , the difference between the 3-year-olds' mean scores and those of the other age groups was significant $(p<.01)$. The 4 - and 5-year-olds groups did not significantly differ from each other. But the comparisons revealed that the age groups did not differ when option 2 was the correct choice.

This experiment confirmed that word order of forcedchoice questions influences children's responses. As in experiment 1 , the results in this experiment revealed that younger children exhibited a stronger tendency toward the second options embedded in questions and showed further that this tendency grew weaker as children aged.

\section{GENERAL DISCUSSION}

In the present study, two experiments were conducted to investigate children's response tendency toward two-option, forced-choice questions, and whether age had an effect on children's answers. Although there were minor discrepancies in the findings, both experiments produced highly consistent results. Overall, it was found that preschoolers' responses are influenced by the way questions are formulated and the way the constituents of sentences are arranged. In the questions tested in this study, young children demonstrated a 'recency tendency' in their responses and appeared to be highly susceptible to minor changes in the structure of questions.

The results of this study are in contrast with the findings reported by Peterson and Grant (2001) although they are consistent with the findings of Fritzley et al. (2009) as well as Rocha et al. (2013). One possible explanation for these inconsistencies may be methodological differences. Children in the current study watched a video clip and then answered questions about it, and those in Fritzley et al.'s study answered questions about familiar and unfamiliar objects. Both studies found strong recency biases in two-option forced-choice questions. Neither of these involved children's personal participation in salient events, and other research has suggested that personal involvement may be important in children's response accuracy (Brown et al., 2012; 2013; Rudy \& Goodman, 1991). In contrast, children in Peterson and Grant's study (Peterson \& Grant, 2001) were individually involved in enacted events, and children in Rocha et al. (2013) were questioned about their dental experiences. Thus, both studies involved personal experience, but the events about which children were questioned differed significantly. In Rocha et al.'s study, the events were highly salient (and even sometimes painful or stressful) as well as chronologically coherent, and such properties of events help children's recall of them (Brown et al., 2012; 2013; Peterson, 2002). These two studies thus differed in the salience of the events in which children participated, and Rocha's study participants probably had discussions with their parents and often dental personnel about their dental experiences before, during, and after the events occurred, something that was unlikely with Peterson and Grant's study participants. Such discussions also may impact children's memory (Conroy \& Salmon, 2006).

Another important consideration is the age of the child participants. The strongest recency tendency in the present study, which investigated 3- to 5-year-olds, was found for 3-year-olds. When looking at 2- to 5-year-olds' responses to familiar and comprehensible forced-choice questions, Fritzley et al. (2009) found the strongest recency tendency by 2 -year-olds, followed by the 3 -year-olds. The smaller response bias found by Rocha et al. (2013) may be because their youngest age group included children between 4 and 6 years of age (mean age $=5.8$ years). Peterson and Grant (2001), whose study participants only included 3- to 4year-olds, were the only ones who did not find an age effect. Overall, the results of this study concerning the 3-year-olds' responses support the previously reported conclusion that children at the age of 3 are at a developmental transition period (Fritzley \& Lee, 2003; Steffensen, 1978). Although this conclusion is based on studies dealing with children's accuracy of response to other types of questions, the results of both experiments in this study revealed that 3-year-old children's response scores are significantly different from 4- and 5-year-olds. It appears that this difference may suggest a genuine developmental phenomenon. One possibility might be that the 3-year-olds may undergo a transition in terms of response tendency.

Another methodological difference between this study and both Peterson and Grant's (2001) and Rocha et al.'s studies is that the latter both involved a delay. Peterson and Grant's participants were interviewed for the first time 1 week after the target events, and Rocha et al.'s participants were interviewed both immediately after and 6-8 weeks later. Rocha et al. found that response biases were worse in their follow-up interview and interpreted poorer performance over 
time as consistent with fuzzy trace theory's proposals about verbatim or detailed memory being more vulnerable to forgetting than gist memories. Recency effects were smaller when interviews were not delayed, and their findings were thus more similar to those of Peterson and Grant in the immediate interview than in the delayed one, although their results are still consistent with a response bias toward the second of two options in forced-choice questions. Other studies suggest that a delay can influence the accuracy of children's responses. For example, Brown et al. (2012) investigated 5- to 7-year-olds children's responses to questions about experienced events both 1 and 7 months following the event. They found that children reported much more information at 7 months, but their responses included a high portion of inaccurate information. In addition, children seldom repeated information across the two interviews and did not incorporate inaccurate information they reported in the first interview into their responses 6 months later. In short, no clear understanding of discrepancies between studies can yet be discerned by considering methodological variation. Further research is needed to clarify the conditions under which response biases occur.

It is also possible that part of this inconsistency between studies is rooted in a linguistic difference. In English, normally a rising intonation is used in the first part of forcedchoice questions, and a falling intonation is used for the second part. For example, 'Would you like an $\nearrow$ apple or a $\searrow$ pear?' This intonation pattern makes the first part of the question a marked choice and thus more prominent for the interviewee. In the questions asked in this study, a different intonation pattern was followed as the questions were asked in Persian. In fact, a rising intonation is often associated with both parts of forced-choice questions in Persian. Therefore, while the specific intonation pattern of forced-choice questions in English may make the first part of the question a marked choice, in Persian, both parts often receive the same level of emphasis. Therefore, when interviewees are asked forced-choice questions in Persian, they are not influenced by the linguistic features of questions in the way an English speaker might be. However, two studies conducted in English (Fritzley et al., 2009, and Rocha et al., 2013) found response biases favoring the second of the options given, which is inconsistent with suggestions that linguistic features partly influence option choice. However, researchers who are specifically studying how children answer forced-choice questions may use question intonation patterns that are not typical of questions in normal speech interactions, and these may play a role in how children respond to forced-choice questions. To see if atypical intonation patterns are used, analysis of intonation patterns would be necessary.

Overall, an important question is whether children in different cultures and who speak different languages show different tendencies in response to forced-choice questions. This may partly be due to the linguistic properties of questions, and future research needs to explore this because such properties may be an influential factor in children's responses. Ceci and Bruck (2006) have argued that social, cognitive, and biological factors seem to play a role in how children respond to questions, and both language and cultural differences must be considered as well. Considering the importance of understanding the influences that can bias children's responses to questions of different types as well as their suggestibility, an important direction for future studies is, therefore, to conduct comparative studies in different languages and cultures. Okanda and Itakura (2007, 2008) have shown that these play an important role in how children respond to yes/no questions, and the current study is a beginning in this direction for two-option questions.

It should be admitted that the present study does not represent a forensic interview nor a medical situation. Rather, the process of interviewing was conducted in children's care center, which was not at all distressing. Rapport was established between the interviewer and participants, and children were not questioned aggressively. All these affective and contextual factors might influence children's responses. There are a number of other limitations of this research as well. The children were not questioned about events that they had personally experienced or that were particularly salient to them, which may well have affected their responses (Brown et al., 2012; 2013; Peterson, 2002). Thus, it is not clear how much attention children paid to the video or how well they remembered the depicted story, although the pilot study showed that they understood the story and were familiar with the animal characters in it. Future research could profit from including controls to ensure that children this age indeed accurately remember the details of the story. In addition, although children had been informed that they could say 'I don't know' if they did not know the answers to the questions, they were not rigorously instructed to answer only when they were sure and not to guess. However, Peterson and Grant (2001) found that repeated reminders of the 'I don't know' option had little effect. It would also be useful for future research to compare children's responses to two-option forced-choice questions with responses to yes/no questions and to include questions where none of the options are correct, as was performed by Rocha et al. (2013).

Nevertheless, this study has implications for designing developmental studies as well as for conducting interviews with children. Researchers and interviewers often have mixed feelings about asking forced-choice questions (Ceci \& Bruck, 1993; Howie et al., 2009; Mehrani, 2011). On the one hand, this type of question can be used to elicit information where other types of questions may not be effective. On the other hand, there is concern that children's responses to forcedchoice questions may not be accurate. The findings of the present study demonstrate that, when interviewing preschoolers, this type of question is not appropriate for use and should ideally be avoided, because children's recency tendency can result in their responses being biased.

This study also illustrates the benefits of empirical investigation into the reliability and effectiveness of questioning as a data collection method in developmental research. In fact, questioning is one of the most commonly used methods for collecting data in developmental investigations. Fritzley and Lee (2003) reported that in $74 \%$ of the developmental studies published between 1995 and 1998 in Child Development and Developmental Psychology, questioning was used as a data collection method. However, there seems to be a paucity of research devoted to the empirical investigation into how questioning is used for investigating developmental 
phenomena (Fritzley \& Lee, 2003). It is crucial that not only question format be explored but that linguistic and cultural differences be considered as well.

In conclusion, this study found evidence of response bias in Persian-speaking 3-year-olds when answering two-option forced-choice questions. Such a bias has also been found in a couple of studies conducted with English-speaking children (although not in all). It is important that children with different language and cultural backgrounds be tested to see if the recency tendency found here is a cross-linguistic and universal phenomenon in young children.

\section{REFERENCES}

Brown, D. A., Lamb, M. E., Lewis, C., Pipe, M., Orbach, Y., \& Wolfman, M. (2013). The NICHD investigative interview protocol: An analogue study. Journal of Experimental Psychology: Applied, 19, 367-382. doi: 10.1037/a0035143

Brown, D. A., Lewis, C., Lamb, M. E., \& Stephens, E. (2012). The influences of delay and severity of intellectual disability on event memory in children. Journal of Consulting and Clinical Psychology, 80, 829-841. doi: $100.1037 / \mathrm{a} 0029388$

Ceci, S. J., \& Bruck, M. (1993). Suggestibility of the child witness: A historical review and synthesis. Psychological Bulletin, 113, 403-439. doi: 10.1037/0033-2909.113.3.403

Ceci, S. J., \& Bruck, M. (2006). Children's suggestibility: Characteristics and mechanisms. Advances in Child Development and Behavior, 34, 247-281. doi: 10.1016/S0065-2407(06)80009-1

Conroy, R., \& Salmon, K. (2006). Talking about parts of a past experience: The impact of discussion style and event structure on memory for discussed and nondiscussed information. Journal of Experimental Child Psychology, 95, 278-297. doi: 10.1016/j.jecp.2006.06.001

Davies, G., Tarrant, A., \& Flin, R. (2000). The impact of questioning style on the content of investigative interviews with suspected child sexual abuse victim. Psychology Crime and Law, 6, 81-97. doi: 10.1080/ 10683160008410834

Endres, J. (1997). The suggestibility of the child witness: The role of individual differences and their assessment. The Journal of Credibility Assessment and Witness Psychology, 1(2), 44-67.

Fritzley, H. V., \& Lee, K. (2003). Do young children always say yes to yesno questions? A metadevelopmental study of the affirmation bias. Child Development, 74(5), 1297-1313. doi: 10.1111/1467-8624.00608

Fritzley, V. H., Lindsay, R. C. L., \& Lee, K. (2009). Is it A) primacy bias or B) recency bias? Young children's response tendencies toward dual-option multiple-choice questions. Presented at the biennial meetings of the Society for Research in Child Development, Denver, CO, April.

Goodman, G. S., \& Quas, J. A. (2008). Repeated interviews and children's memory: It's more than just how many. Current Directions in Psychological Science, 17, 386-390. doi: 10.1111/j.1467-8721.2008.00611.x

Goodman, G. S., \& Schaaf, J. M. (1997). Over a decade of research on children's eyewitness testimony: What have we learned? Where do we go from here? Applied Cognitive Psychology, 11, S5-S20. doi: 10.1002/ (SICI)1099-0720(199712)11:7,S5::AID-ACP545>3.0.CO;2-C

Howie, P., Kurukulasuriya, N., Nash, L., \& Marsh, A. (2009). Inconsistencies in children's recall of witnessed events: The role of age, question format and perceived reason for question repetition. Legal and Criminological Psychology, 14, 311-329. doi: 10.1348/135532508X383879

Howie, P., Sheehan, M., Mojarrad, T., \& Wrzesinska, M. (2004). 'Undesirable' and 'desirable' shifts in children's responses to repeated questions: Age differences in the effect of providing a rationale for repetition. Applied Cognitive Psychology, 18, 1161-1180. doi: 10.1002/acp.1049

Lamb, M. E., Orbach, Y., Hershkowitz, I., Esplin, P., \& Horowitz, D. (2007). A structured forensic interview protocol improves the quality and informativeness of investigative interviews with children: A review of research using the NICHD Investigative Interview Protocol. Child Abuse \& Neglect, 31, 1201-1231. doi: 10.1016/chiabu.2007.03.021
Lamb, M. E., Sternberg, K. J., Orbach, Y., Esplin, P. W., Stewart, H., \& Mitchell, S. (2003). Age differences in young children's responses to open-ended invitations in the course of forensic interviews. Journal of Consulting and Clinical Psychology, 71, 926-934. doi: 10.1037/0022006X.71.5.926

Larsson, A. S., \& Lamb, M. E. (2009). Making the most of informationgathering interviews with children. Infant \& Child Development, 18, 1-16. doi: 10.1002/ICD.573

Mehrani, M. B. (2011). What is biased? Children's strategies or the structure of yes/no questions? First Language, 31, 214-221. doi: 10.1177/ 0142723710371886

Mehrani, M. B., \& Peterson, C. (2014). Interviewing preschoolers: Responses to yes/no questions. Manuscript submitted for publication.

Newcombe, P. A., \& Dour, T. M. (2001). Conversational influences on young children's responses to misleading questions. Applied Developmental Psychology, 22, 363-378. doi: 10.1016/S0193-3973(01)00078.8

Okanda, M., \& Itakura, S. (2007). Do Japanese children say 'yes' to their mothers? A naturalistic study of response bias in parent-toddler conversations. First Language, 27, 421-429. doi: 10.1177/ 0142723707081653

Okanda, M., \& Itakura, S. (2008). Children in Asian cultures say yes to yesno questions: Common and cultural differences between Vietnamese and Japanese children. International Journal of Behavioural Development, 32, 131-136. doi: 10.1177/0165025407087211

Okanda, M., \& Itakura, S. (2010). When do children exhibit a "yes" bias? Child Development, 81, 568-580. doi: 10.1111/j.1467-8624.2009

Peterson, C. (2002). Children's long-term memory for autobiographical events. Developmental Review, 22, 370-402. doi: 10.137/00121649.35.6.1493

Peterson, C. (2012). Children's autobiographical memories across the years: Forensic implications of childhood amnesia and eyewitness memory for stressful events. Developmental Review, 32, 287-306. doi: 10.1002/ acp. 1636

Peterson, C., \& Biggs, M. (1997). Interviewing children about trauma: Problems with "specific" questions. Journnal of Traumatic Sterss, 10, 279-290. doi: 10.1023/A:1024882213462

Peterson, C., Dowden, C., \& Tobin, J. (1999). Interviewing preschoolers: Comparison of yes/no and wh-questions. Law and Human Behavior, 23, 539-555. doi: 10.1023/A:1022396112719

Peterson, C., \& Grant, M. (2001). Forced-choice: Are forensic interviewers asking the right questions? Canadian Journal of Behavioural Science, 33, 118-127. doi: 10.1037/h0087134

Pipe, M. E., Thierry, K. S., \& Lamb, M. E. (2006). The development of event memory: Implications for child witness testimony. In M. P. Toglia, J. D. Read, D. F. Ross, \& R. C. L. Lindsay (Eds.), Handbook of eyewitness psychology, Vol. 1: Memory for events (pp. 447-472). Mahwah, New Jersey: Lawrence Erlbaum Associates.

Poole, D., White, L. (1991). Effects of question repetition on the eyewitness testimony of children and adults. Developmental Psychology, 27, 975-986. doi: 10.1037/0012-1649.27.6.975

Powell, M. B., Fisher, R. P., \& Wright, R. (2005). Investigative interviewing. In N. Brewer, \& K. D. Williams (Eds.), Psychology and law: An empirical perspective (pp. 11-42). New York: Guilford Press.

Quas, J. A., Goodman, G. S., Ghetti, S., \& Redlich, A. (2000). Questioning the child witness. What can we conclude from the research this far? Trauma, Violence and Abuse, 1, 223-249. doi: 10.1177/ 1524838000001003002

Reyna, V. F., Holliday, R., \& Marche, T. (2002). Explaining the development of false memories. Developmental Review, 22, 436-489. doi: 10.1016/S0273-2297(02)00005-5

Rocha, E. M., Marche, T. A., \& Briere, J. L. (2013). The effect of forcedchoice questions on children's suggestibility: A comparison of multiplechoice and yes/no questions. Canadian Journal of Behavioural Science, 45, 1-11. doi: 10.1037/a0028507

Rudy, L., \& Goodman, G. S. (1991). Effects of participation on children's report: Implications for children's testimony. Developmental Psychology, 27, 527-538. doi: 10.1037/0012-1649.27.4.527

Steffensen, M. S. (1978). Satisfying inquisitive adults: Some simple methods of answering yes/no questions. Child Language, 5, 221-236. doi: 10.1017/S0305000900007431 